\title{
Effect of Sleeping Instruction on Reducing Insomnia among Elderly
}

\section{Aisha saad eldein saleh ${ }^{1}$, Amal Mohamed Ahmed ${ }^{2}$, Hend Elham Mohamed ${ }^{3}$}

1. Clinical instructor of Geriatric Nursing Department,Faculty of Nursing - Aswan University.

2. Professor\& head of Medical Surgical Nursing, Faculty of Nursing - Aswan University

3. Assistant prof of Medical Surgical Nursing, Faculty of Nursing -Minia University.

*Email of the corresponding author: zahrt.elnoba@yahoo.com

\begin{abstract}
Background: Insomnia disorder is a public health concern and is often considered a normal aging phenomenon. Aim of the study: evaluating the effect of sleeping instruction on reducing insomnia among the elderly. Design: A quasiexperimental design. Settings: conducted at sleep disturbance outpatient clinic in Aswan university hospital \&geriatric social club in Aswan city. Sample: A purposive sample was used to conduct this study, the sample size was 60, and they were chosen according to inclusion criteria. Tools: Two tools were used in the present study; a structured interview questionnaire \& Athens insomnia scale Results: The designed sleep hygiene program had statistically significant improvement in elderly knowledge and practices. Indeed, there was no statistically significant correlation between elderly' sleep hygiene and Athens insomnia scale as sleep latency, sleep duration, sleep efficiency, sleep disturbances, and day time dysfunction at the pre and post-intervention phases at $\mathrm{P}<$ 0.05.Conclusion: The designed sleep hygiene program don in this study had statistically significant improvement in elderly knowledge and practices and reducing insomnia. Recommendations: Design an awareness Program regarding insomnia among the elderly to enhance sleep by emphasizing the importance of sleep hygiene practice.
\end{abstract}

Keywords: Insomnia, Older adult, Sleep Hygiene Program.

\section{Introduction}

Sleep is a vital physiological process with important restorative functions, and plays a role in biological function of central importance for most living things (Tucker, 2020). Studies on the physiology of sleep have shown that a variety of highly relevant processes take place during sleep, such as energy conservation, metabolic regulation, memory consolidation, elimination of waste substances, activation of the immune system. (Jha V \& Jha S., 2020).

With age, several changes occur that can place one at risk for sleep disturbances including increased prevalence of medical and psychiatric comorbidities, increased medications use, age related changes in various circadian rhythms, and environmental and life style changes (Heidari \& Huege., 2020). One of the major changes that commonly accompany the aging process is an often profound disruption of an individual's daily sleep-wake cycle lead to sleep impairments (Jurado-Fasoli, et al., 2020).

Insomnia disorder is an economic burden and public health concern. It is mostly seen in older age groups, and often considered a normal aging phenomenon (Freeman, et al., 2020). Many studies have suggested the etiology of insomnia may be related to many factors such as genetics, biochemistry, neuroendocrine, immune, and psychosocial factors $(\mathbf{L i}, \mathbf{Y}$ et al., 2020).Insomnia impairs the individual functioning and diminishes the quality of life, as it associated with low score of mental and physical component summary of quality of life (Gothe, et al .,2020) .

Sleep hygiene is non-pharmacologic measures to increase the quantity and quality of clients 'sleep. Sleep hygiene encompasses health education on sleep rituals, habits and restful environment. Current research suggests that nonpharmacological interventions, specifically, changes made to the environment, could potentially increase the sleep quality of geriatric patients (Harrelson ., 2017).

$P$ a g e $\mid 65$
Nurses are expected to be aware of their possible causes, in order to early recognize them and support the proposal of interventions to minimize them. From a nursing perspective, insomnia can be considered a human response to a health condition or a life process, i.e., a nursing diagnosis. The NANDA International, Inc. (NANDA-I) Diagnosis Classification defines Insomnia as a disruption in amount and quality of sleep that impairs functioning (Guandalini, et al., 2020).

It is essential that healthcare professionals understand the normal distribution of the sleep stages to understand changes that occur to sleep related to disease, cognitive changes, and reactions to medication. Nurses should remember that numerous factors can contribute to sleep stage distribution and circadian cycle disruptions interrelated factors must be considered, including medical, psychiatric, and cognitive comorbidities, as well as the use of sedatives and hypnotics. (Hedges, \& Gotelli., 2020).

\section{Significance of the study:}

Sleep disorders are relatively poorly undiagnosed and untreated despite poor sleep strongly affects the daily lives of millions of people around the world. Insomnia can cause significant morbidity if not addressed properly. (Richter,et al .2020). The increase in the aged population will bring with it a huge burden of sleep related health problems. Although ageing is a global phenomenon, little data are available on regional trends in sleep related problems (WHO, 2019).

Hence, the present study conducted to reducing Insomnia among older adults attending the setting of study. Insomnia is highly prevalent, especially at advanced age. It is a high societal, medical cost and associated with increased health service utilization. (Timalsina, 2017). Therefore, awareness regarding insomnia and sleep hygiene education among elderly should be raised for early detection and 
appropriate management of insomnia in order to decrease sleep related health problems. (Chhantyal \& Timalsina, 2017).

Aim of the study:

Evaluating the effect of sleeping instruction on reducing insomnia among elderly.

\section{Subjects and Methods \\ Research Design:}

A quasi-experimental design was used to achieve the aims of the study.

\section{Subjects (sample):}

Purposive sample of (60) elderly, their age ranged between 65-75years that are diagnosed with insomnia.

For the sample size $n$, this calculator employs the following formula:

$$
\begin{gathered}
n=N * X /(X+N-1), \\
\text { Where, } \\
X=Z_{\alpha / 2}^{2} * p *(1-p) / M^{2} E^{2},
\end{gathered}
$$

And $\mathrm{Z} / 2$ is the critical value of the Normal distribution at $/ 2$ (for example, for a 95percent confidence level, is 0.05 and the critical value is 1.96), MOE is the margin of error, $\mathrm{p}$ is the sample proportion, and $\mathrm{N}$ is the participant scale. The sample size formula $(n=60)$ has been subjected to a Finite Population Correction.

\section{Inclusion criteria:}

- Newly diagnosed elderly with insomnia age eligible for study 65 years to 75 years.

- Male and female regardless education level.

\section{Exclusion criteria:}

- Receiving Drugs that effect on sleep.

- Suffering with Mental or Psychological disorder.

- Refuse to participate.

\section{Setting:}

This study was carried out at sleep disturbance outpatient clinic in Aswan University Hospital \&Geriatric Social Club in Aswan City.

\section{Study Duration:}

The fieldwork and data collection for this study lasted three months using the data collection tools, from the beginning of June 2018 till the end of august 2018.

\section{Tools of data collection:}

Two tools were utilized to collect pertinent data for study includes:

\section{First Tool:}

A structured interview questionnaire sheet was developed by researcher after revising extensive literature review. (Abd Allah, et al., 2014) (Abd Allah, et al., 2016) (El -Gilany, et al., 2017). It was collected before, after and follows up from educational instruction and it consists of two parts:

$1^{\text {st }}$ part: Demographic characteristics of the study elderly: Includes data such as; age, sex, educational level, current occupation, residence, marital status, income, income source. $2^{\text {nd }}$ part: Medical data:

It was involve questions about chronic disease, medications regimen, and day time problems caused by inadequate sleep, habits before Sleeping and environmental factors affect sleep.

Second Tool: Athens insomnia scale (AIS): This scale was developed by (Soldatos et al, 2003).

The scale is a useful screening tool in reliably establishing the diagnosis of insomnia. This scale used to evaluate insomnia through a detailed sleeping history during the last month. It consists of eight items. The first five items cover night-time symptoms of insomnia (difficulty initiating sleep, difficulty maintaining sleep and early morning awakening, total sleep period, and overall quality of sleep), while the last three items ask for daytime consequences of disturbed sleep (problems with sense of well-being, overall functioning, and sleepiness during the day).

Scoring system: The eight items of the AIS were rated from zero to 3 , (with 0 corresponding to no problem at all and 3 to very serious problem. The elderly subjects are asked to grade the severity of these complaints (absent $=0$, mild $=1$, severe $=2$, and very severe $=3$ ). Elderly subject who had a total score 6 points or higher was considered as a positive case for insomnia.

Educational Booklet to enhance effective Sleeping Practice among Elderly: The researcher prepared Educational Booklet to enhance effective Sleeping Practice among elderly. The booklet information based on review of relevant literature (Melinda Smith, et al., 2020) about insomnia, habits and environmental factors that interfering with sleep and how to avoid these factors. This booklet was reviewed by supervisors.

\section{Tools validity and Reliability:}

The tools were tested for content validity by assent of five expertises from the faculty of nursing (a jury of five expert's opinion). The tool was tested for internal reliability by using Cronbach's alpha. The test-retest reliability correlation coefficient found almost 0.90 at a 1-week interval. As far as external validity is concerned, the correlations of the AIS-8 and AIS-5 with the Sleep Problems Scale were 0.90 and 0.85 , respectively.

\section{Pilot Study:}

A pilot study conducted on $10 \%$ of the total study sample represent 6 person from study sample. In order to test clarity, applicability and feasibility of the tools.

\section{Ethical Consideration:}

An official permission and administration permission to conduct the study was obtained; Approval of participating cases was taken through Oral consents. Elderly were informed about the purpose, procedure, benefits, nature of the study; follow up and his/her had the right to withdraw from the study at any time. Strict Confidentiality and anonymity of each and Informed them that obtained data will not be included at any further researches without a second consent.

\section{Study Procedure:}

The researcher has started to collection data used an interview questionnaire sheet (First tool) and (Second tool). Collection of study data was done two days/week. The researcher was interviewing the patients individually and explained the purpose of the study briefly and nature of study 
to the studied elderly. The intervention was offered to the studied sample in the form of sessions ( 5 sessions) for small group the time consumed for answering the study tools ranged from 30 to 45 minutes. The total sample was divided into small groups (5 elderly in each group). All groups received the same content using the same teaching methods, media, discussions, and the same booklet. Follow up; after one month from giving instructions.

\section{Limitation of the study:}

- Time available for follow up not enough as many patients.
- Participants suffered from transportation and difficulty of mobility due to health status, physical condition and financial problems.

\section{Statistical analysis:}

The collected data organized categorized, tabulated and analyzed using SPSS ver. 24. Data presented in tables and charts using numbers and percentage, statistics and associations done using mean, standard deviation SD, t- test and p- value, Significant of the result: non significance $(\mathrm{P}>0.05), *$ : Significance $(\mathrm{p}<0.05) * *$ : moderate significance $(\mathrm{P}<0.001) * * *$ : highly significance $(\mathrm{P}<0.000)$.

\section{Results and data analysis:}

Table (1): Relation between insomnia \&demographic data in study sample pre educational protocol

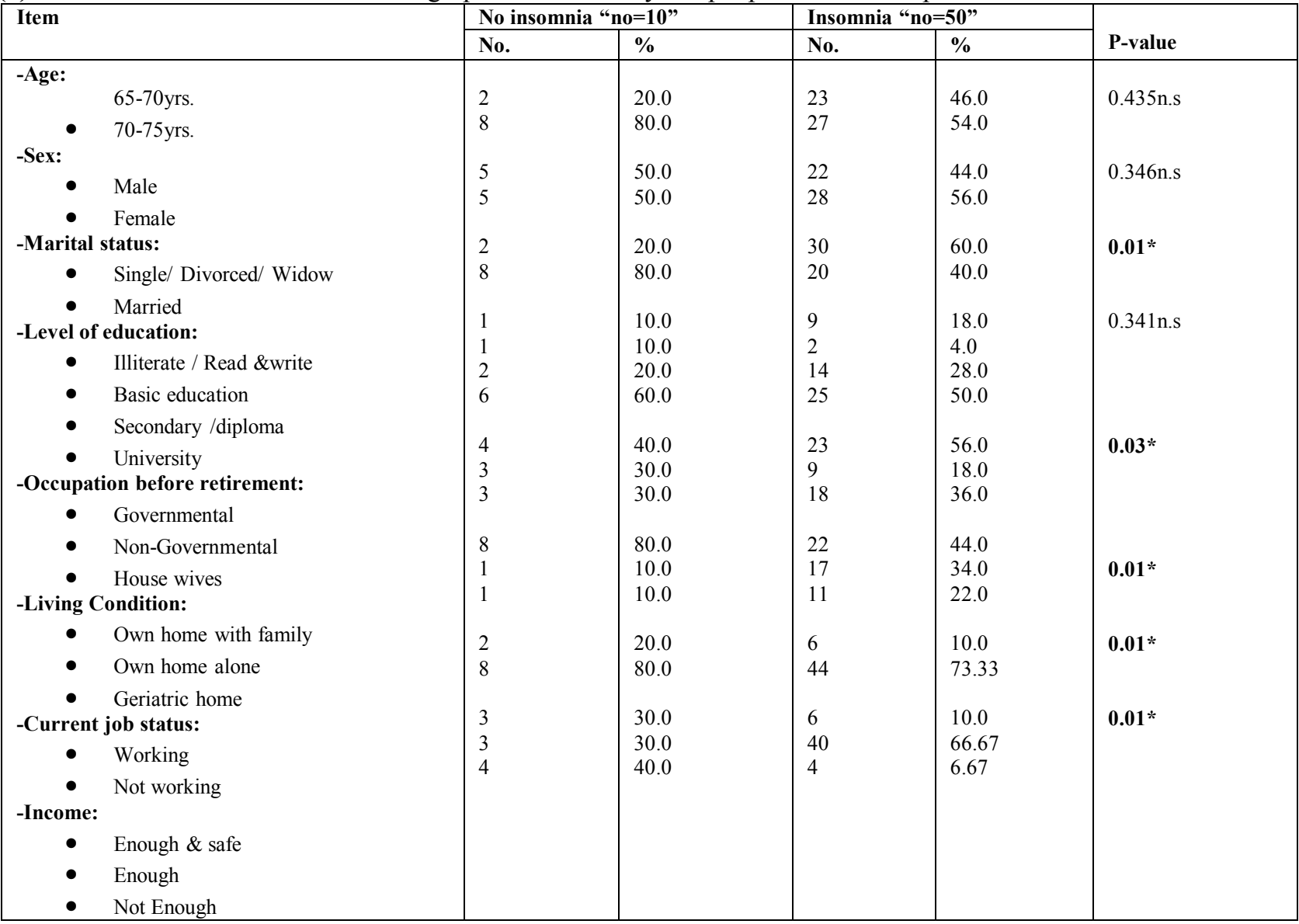
$(\mathrm{P}<0.000)$.

N.S: non significance $(\mathrm{P}>0.05), *$ : Significance $(\mathrm{p}<0.05) * *$ : moderate significance $(\mathrm{P}<0.001) * * *$ : highly significance

The table (1) Demonstrated relation between insomnia \&demographic data in study sample pre educational protocol. There was no statistical significance difference between age, sex and level of education with insomnia at $(\mathrm{P}>0.05)$, while there was statistical significance difference between Marital status, occupation before retirement, living condition, current job status and income with insomnia at $(\mathrm{P}<0.05)$.

Table (2): Relation between insomnia and medical history data in study sample.

\begin{tabular}{|l|c|c|c|c|c|}
\hline \multirow{2}{*}{ Item } & \multicolumn{2}{|c|}{ No insomnia “n=10" } & \multicolumn{2}{c|}{ Insomnia “n=50" } & P-value \\
\cline { 2 - 6 } & No. & $\mathbf{\%}$ & No. & \% & \\
\hline - Hypertension & 4 & 40.0 & 27 & 54.0 & $0.342 \mathrm{n} . \mathrm{s}$ \\
-Orthopedic disease & 2 & 20.0 & 24 & 48.0 & $0.02 *$ \\
-Diabetes & 5 & 50.0 & 23 & 46.0 & $0.375 \mathrm{n} . \mathrm{s}$ \\
-Cardiac disease & 2 & 20.0 & 6 & 12.0 & $0.439 \mathrm{n} . \mathrm{s}$ \\
-Hepatic disease & 0 & 0 & 3 & 6.0 & $0.246 \mathrm{n} . \mathrm{s}$ \\
-GIT disease & 1 & 10.0 & 6 & 12.0 & $0.473 \mathrm{n} . \mathrm{s}$ \\
-Chest disease & 0 & 0 & 17 & 28.0 & $0.01 *$ \\
-Renal disease & 0 & 0 & 4 & 8.0 & $0.342 \mathrm{n} . \mathrm{s}$ \\
-Neuro disease & 1 & 10.0 & 3 & 6.0 & $0.273 \mathrm{n} . \mathrm{s}$ \\
-Endocrine & 1 & 10.0 & 2 & 4.0 & $0.342 \mathrm{n} . \mathrm{s}$ \\
\hline
\end{tabular}


N.S: non significance $(\mathrm{P}>0.05)$, *: Significance $(\mathrm{p}<0.05) * *$ : moderate significance $(\mathrm{P}<0.001) * * *$ : highly significance $(\mathrm{P}<0.000)$.

Table (2) illustrated relation between insomnia and medical history data in study sample. There was statistical significance difference between orthopedics and chest disease with insomnia at $(\mathrm{P}<0.05)$.

Table (3): Relation between Habits before sleeping and insomnia in study sample.

\begin{tabular}{|l|c|c|c|c|c|}
\hline \multirow{2}{*}{ Item } & \multicolumn{2}{|c|}{ No insomnia "n=10" } & \multicolumn{2}{|c|}{ Insomnia "n=50" } & \multirow{2}{*}{ P-value } \\
\cline { 2 - 5 } & No. & $\mathbf{\%}$ & No. & \% & \\
\hline Good habits: & & & & & \\
-Exercise regularly during day & 0 & 0 & 2 & 4.0 & $0.438 \mathrm{n} . \mathrm{s}$ \\
-Relax before bed time & 4 & 40.0 & 4 & 6.7 & $0.001^{* *}$ \\
-Establish a regular time for sleep\& getting up & 7 & 70.0 & 10 & 8.0 & $0.001^{* *}$ \\
-Regular waking up early & 6 & 60.0 & 18 & 20.0 & $0.000^{* * *}$ \\
-Eat light meal or snack before bed time & 2 & 20.0 & 6 & 12.0 & \\
-Exposure to light during the day & 8 & 80.0 & 25 & 50.0 & $0.000^{* * *}$ \\
Bad habits: & & & & \\
-Exercising close to bed time & 1 & 0 & 1 & 4 & $0.438 \mathrm{n} . \mathrm{s}$ \\
-Smoking before bed time & 1 & 10.0 & 13 & 26.0 & $0.02^{*}$ \\
-Eating much food before bed time & 1 & 10.0 & 28 & 56.0 & $0.001^{* * *}$ \\
-Going to bed hungry & 3 & 30.0 & 9 & 18.0 & $0.431 \mathrm{n} . \mathrm{s}$ \\
-Drinking caffeinated drink 4hrs. before bedtime & 1 & 10.0 & 39 & 98.0 & $0.000^{* * *}$ \\
-Drinking a lot before bedtime & 1 & 10.0 & 46 & 62.0 & $0.000^{* * *}$ \\
-Napping or resting during day & 3 & 30.0 & 45 & 92.0 & $0.000^{* * *}$ \\
-Watching TV, reading at bed & & 90.0 & $0.001^{* *}$ \\
\hline
\end{tabular}

N.S: non significance $(\mathrm{P}>0.05)$, *: Significance $(\mathrm{p}<0.05) * *:$ moderate significance $(\mathrm{P}<0.001)$ ***: highly significance

Table (3) Showed relation between Habits before sleeping and insomnia in study sample. There was statistical significance difference smoking and insomnia at $(\mathrm{P}<0.05)$. While, there was moderate statistical significance between relax before bed time, establish regular time for sleep and watching TV, reading at bed at $(\mathrm{P}<0.001)$.in addition to there was highly statistical significance difference with each of eating much food before time to bed, drinking caffeinated drink 4 hrs before bedtime, drinking a lot before bedtime, napping or reading at bed and regular waking up early and exposure to light during day at $(\mathrm{P}<0.000)$.

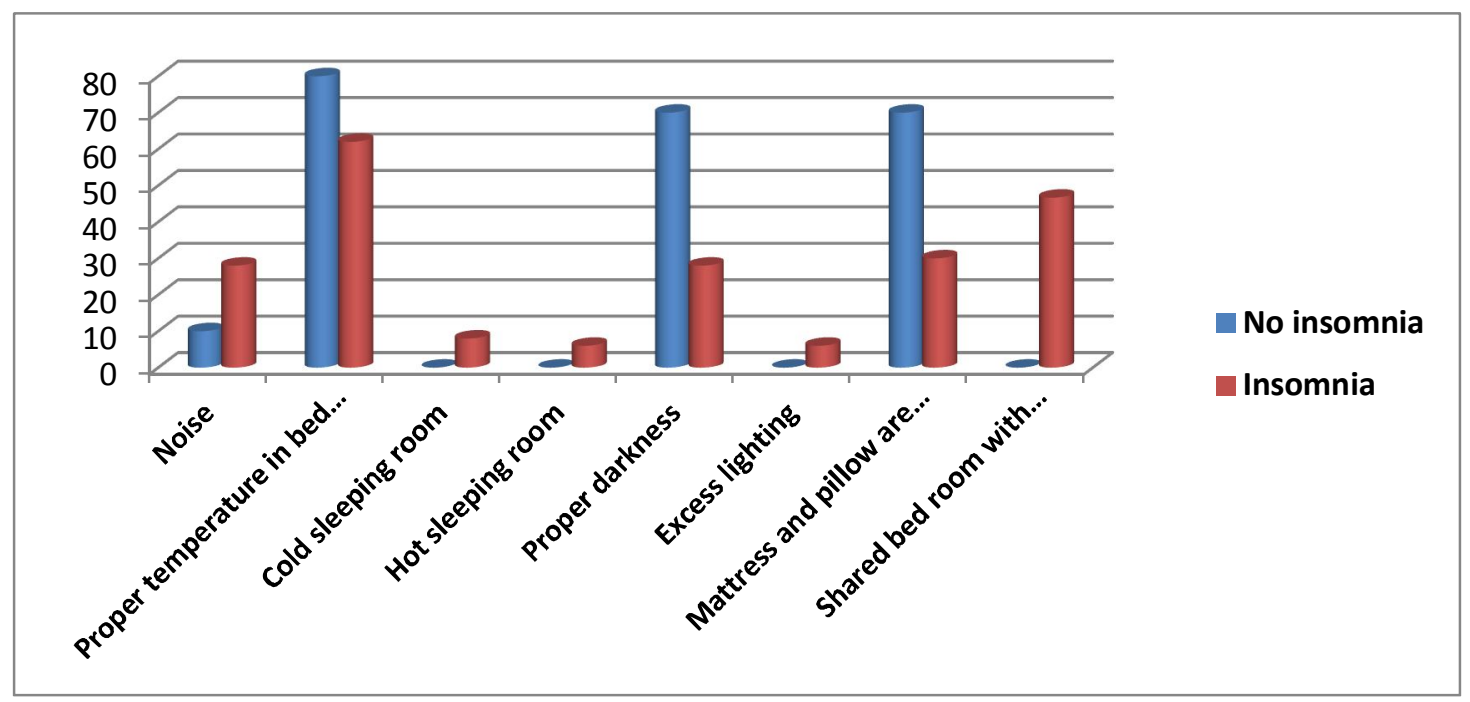

Figure (1): Relation between Environmental factors \& insomnia in study sample.

Fig (1): represented Relation between Environmental factors \& insomnia in study sample. There was statistical significance difference between insomnia with each of proper darkness, mattress and pillow are comfortable and noise at $(\mathrm{P}<0.05)$.

\section{Discussion}

Sleep is a key indicator of quality of life. Therefore, changes that occur in sleep during the aging process affect quality of life negatively. Sleep as long as required to feel rested and then get out of bed. Health practices (e.g., diet, exercise, and substance use) and environmental factors (e.g., light, noise, temperature, and mattress) that may be either detrimental or beneficial to sleep are assessed. (Brewer, 2019).

Insomnia is highly prevalent, it represents the number one sleep disorder encountered in the geriatric clinic population (Roth, et al., 2019), frequently characterized by the subjective complaint of difficulty falling or maintaining sleep, or non-restorative sleep, producing significant daytime symptoms including difficulty concentrating and mood disturbances. (Molzof, et al., 2020).

As regard to the relation between insomnia and demographic data, the current study revealed that there was no statistical significant difference between ages. This is in agreement with Chhantyal \& Timalsina., (2017). In the same direction, Miner, et al., (2018) observed that there is no correlation between age and insomnia. However, according to Shrestha, et al., (2017) and Wang, et al., (2016), there is a correlation between age and insomnia. The lack of agreement in research investigating the correlation between age and insomnia indicates that sleep disturbances can be caused by several psychosocial and biological causes rather than aging. 
Insomnia can be deemed a "multifactorial geriatric disorder," as Korkmaz Aslan, et al., (2020). Recently proposed.

According to the relation between insomnia and sex, the current study finding revealed that there was a non significance difference. This result supports the findings by Dragioti, et al., (2018). However, studies by Kim, et al., (2017) found a relationship between sex (women) and insomnia. This finding is contrary to the study that reported that the female gender was associated with increased risk for insomnia in the Indian older people Ahmad, et al., (2016). We can recommend that sex couldn't be recognized as an independent risk factor for insomnia because being a woman might be accompanied by a low education degree, suffering from poor health, and chronic diseases

In the current study, there was a significant difference $(\mathrm{P}<0.05)$ with no insomnia in married cases. Our findings differed from those of the study done by Dangol, et al., (2020), who found no connection between factors like marital status and insomnia.

The current study found a non-significant association between insomnia and literacy level. Insomnia was nonsignificantly present among the illiterate group of older people than the literate ones, this result disagree with Su, Huang, \& Chou., (2016) and Kim, et al., (2017) In comparison to people with a high education degree, those with a low education rating have a greater risk of developing insomnia. Consistent with previous research Smagula., et al., (2016)

Also, There was a statistical significance difference $(\mathrm{P}<0.05)$ between occupation before retirement, Living Condition, current working status, income, and insomnia. Our results suggest that being active and having a satisfying social life appear to be protective factors against insomnia symptoms at any age. And not engaged in physical activities was the factor associated with insomnia among the elderly. The result is consistent with the Oganbode, et al., (2014) stated that there is a relationship between current employment status and insomnia, as well as a relationship between financial dependence on others and poor salaries, which may lead to insomnia.

In agreement with Ayob, et al., (2014) who reported that physical health complication like pain from orthopedics illness and nocturnal were the factors related with insomnia. This result is the same line as Weronika, et al., (2016) Tsaras, et al., (2021). And we can attribute that to the biological factors that lead to the incidence of insomnia, one of these factors is sleep disorders breathing such as asthma, congestive heart failure, chronic obstructive pulmonary diseases, and sleep apnea. The most factor of insomnia is disorders that cause chronic pain, like arthritis. On the opposite, some researches showed that there is no relationship between the chronic diseases and insomnia Chhantyal, \& Timalsina., (2017).

Concerning with relation between insomnia and habits before sleeping in the study sample, There were statistically significant differences between smoking and insomnia at $(\mathrm{P}<0.05)$. Our findings differed from the study found that older people who had a habit of smoking have no association with insomnia symptoms Shrestha, et al., (2017). While there was moderate statistical significance between relaxation before sleeping, create regular time for sleep, reading, and watching $\mathrm{TV},(\mathrm{P}<0.001)$. in addition to there was a highly statistically significant difference with each of eating a large amount of food before time to bed, drinking caffeine before bedtime, drinking a lot of fluid before bedtime, napping or reading at the bed and regular waking up early and exposure to light during the day $(\mathrm{P}<0.000)$.

Additionally, the routine can help a patient ready for sleeping. This should include discontinuation of noisy activities, including exposure to bright light such as computer screen, which can negatively affect one's circadian rhythms (Andrew, et al., 2019).

\section{Conclusion:}

Based on the findings and research hypothesis of the present study, it can be concluded that: The designed sleep hygiene program don in this study had statistically significant improvement in elderly knowledge and practices and reducing insomnia.

\section{Recommendation:}

- Design awareness (educational) Program regarding insomnia among elderly

- Design educational program among nurses for elders to enhance sleep through emphasizing on the importance of sleep hygiene practice, behavioral and non-pharmacological interventions, and healthy life style.

- Design awareness program regarding maintenance of sleep hygiene for the prevention on large scale places of insomnia should be rose which ultimately leads to improve quality of sleep.

\section{References:}

(1) Abd Allah, E. S., Abdel-Aziz, H. R., \& El-Seoud, A. R. A. (2014). Insomnia: prevalence, risk factors, and its effect on quality of life among elderly in Zagazig City, Egypt. Journal of nursing education and practice, 4(8), 52.

(2) Abd Allah, E. S., Abdel-Aziz, H. R., Ali, S. A., \& ElSeoud, A. R. A. (2016). Cognitive behavioral intervention to improve sleep quality in older adults. IOSR journal of nursing and health science Vol.5, PP 84-94.

(3) Ahmad, E., Ansari, A. H., \& Khan, R. M. (2016). Prevalence of Bekhwabi (Insomnia) among the Elderly Patients Attending Nium Hospital, Bangalore. India. J Community Med Health Educ, 6(476), 2161-0711.

(4) Andrew, D., Krystal1, 2., Aric, A., Prather,1., Liza, H., Ashbrook.(2019). The assessment and management of insomnia: an update. World Psychiatry 18:3 - October 2019.

(5) Ayoub, A. I., Attia, M., El Kady, H. M., \& Ashour, A. (2014). Insomnia among community dwelling elderly in Alexandria, Egypt. The Journal of the Egyptian Public Health Association, 89(3), 136-142.

(6) Brewer, D. (2019). The Science, Techniques and Tips for How to Get To Sleep. Lulu. com.

(7) Chhantyal, A., \& Timalsina, R. (2017). Factors associated with insomnia among elderly of a selected community of Lalitpur. J Gerontol Geriatr Res, 6, 410.

(8) Chhantyal, A., \& Timalsina, R. (2017). Factors associated with insomnia among elderly of a selected community of Lalitpur. J Gerontol Geriatr Res, 6, 410.

(9) Dangol, M., Shrestha, S., \& Rai Koirala, S. K. (2020). Insomnia and its associated factors among older people of selected ward of Banepa municipality, Nepal. Nursing open, 7(1), 355-363.

(10) Dragioti, E., Bernfort, L., Larsson, B., Gerdle, B., \& Levin, L. A. (2018). Association of insomnia severity with well $\square$ being, quality of life and health care costs: A cross $\square$ sectional study in older adults with chronic pain (PainS65+). European Journal of Pain, 22(2), 414-425. 
(11) El-Gilany, A. H., Saleh, N. M. H., Mohamed, H. N. A. E. A., \& Elsayed, E. B. M. (2017). Prevalence of insomnia and its associated factors among rural elderly: a community based study. International Journal of Advanced Nursing Studies, 6(1), 56.

(12) Freeman, D., Sheaves, B., Waite, F., Harvey, A. G., \& Harrison, P. J. (2020). Sleep disturbance and psychiatric disorders. The Lancet Psychiatry, 7(7), 628-637.

(13) Gothe, N. P., Ehlers, D. K., Salerno, E. A., Fanning, J., Kramer, A. F., \& McAuley, E. (2020). Physical activity, sleep and quality of life in older adults: Influence of physical, mental and social well-being. Behavioral sleep medicine, 18(6), 797-808.

(14) Guandalini, L. S., da Silva, E. F., de Lima Lopes, J., Santos, V. B., Lopes, C. T., \& de Barros, A. L. B. L. (2020). Analysis of the evidence of related factors, associated conditions and at-risk populations of the NANDA-I nursing diagnosis insomnia. International journal of nursing sciences.

(15) Harrelson, C. M. (2017). Sleep Quality Effects of NonPharmacological Protocol in Community Based Geriatric Population. Gardner-Webb University.

(16) Hedges, C., \& Gotelli, J. (2020). Managing insomnia in older adults. Nursing2020, 50(9), 24-32.

(17) Heidari, S., \& Huege, S. (2020). Sleep Disorders in the Geriatric Population. In Sleep Medicine and Mental Health (pp. 293-313). Springer, Cham.

(18) Jha, V. M., \& Jha, S. K. (2020). Sleep: Basic and Historical Aspects. In Sleep: Evolution and Functions (pp. 1-15). Springer, Singapore.

(19) Jurado-Fasoli, L., Mochon-Benguigui, S., Castillo, M. J., \& Amaro-Gahete, F. J. (2020). Association between sleep quality and time with energy metabolism in sedentary adults. Scientific reports, 10(1), 1-10.

(20) Kim, S. A., Koo, B. B., Kim, D. E., Hwangbo, Y., \& Yang, K. I. (2017). Factors affecting fatigue severity in patients with obstructive sleep apnea. The clinical respiratory journal, 11(6), 1045-1051.

(21) Kim, W. J., Joo, W. T., Baek, J., Sohn, S. Y., Namkoong, K., Youm, Y., \& Lee, E. (2017). Factors associated with insomnia among the elderly in a Korean rural community. Psychiatry investigation, 14(4), 400.

(22) Korkmaz Aslan, G., İncİ, F. H., \& Kartal, A. (2020). The prevalence of insomnia and its risk factors among older adults in a city in Turkey's Aegean Region. Psychogeriatrics, 20(1), 111-117.

(23) Li, Y., Zhang, B., Zhou, Y., Wang, D., Liu, X., Li, L., \& Hoven, C. W. (2020). Gut Microbiota Changes and Their Relationship with Inflammation in Patients with Acute and Chronic Insomnia. Nature and Science of Sleep, 12, 895.

(24) Melinda Smith, M.A., Lawrence Robinson., Robert Segal, M.A., (2020). Sleep Tips for Older Adults. Help Guide organization.

(25) Miner, B., Gill, T. M., Yaggi, H. K., Redeker, N. S., Van Ness, P. H., Han, L., \& Fragoso, C. A. V. (2018). Insomnia in community $\square$ living persons with advanced age. Journal of the American Geriatrics Society, 66(8), 1592-1597.
(26) Molzof, H. E., Petrov, M. E., \& Lichstein, K. L. (2020). Late-Life Insomnia. In Geriatric Practice (pp. 267-278). Springer, Cham.

(27) Ogunbode, A. M., Adebusoye, L. A., Olowookere, O. O., Owolabi, M., \& Ogunniyi, A. (2014). Factors associated with insomnia among elderly patients attending a geriatric centre in Nigeria. Current gerontology and geriatrics research, 2014.

(28) Richter, K., Kellner, S., Milosheva, L., \& Fronhofen, H. (2020). Treatment of insomnia in elderly patients. Journal for ReAttach Therapy and Developmental Diversities, 2(2), 129-138.

(29) Roth, T., Coulouvrat, C., Hajak, G., et al. (2019). Prevalence and perceived health associated with insomnia based on DSM-IV-TR; International Statistical Classification of Diseases and Related Health Problems, Tenth Revision; and Research Diagnostic Criteria/International Classification of Sleep Disorders, Second Edition criteria: results from the America Insomnia Survey. Biol Psychiatry. 2019; 69(6):592-600

(30) Shrestha, S., Roka, T., Shrestha, S., \& Shakya, S. (2017). Prevalence and contributing factors of insomnia among elderly of Pashupati old aged home (Briddhashram). Mathews Journal of Psychiatry \& Mental Health, 2(2), 014.

(31) Smagula, S. F., Stone, K. L., Fabio, A., \& Cauley, J. A. (2016). Risk factors for sleep disturbances in older adults: evidence from prospective studies. Sleep medicinereviews, 25,21-30.

(32) Soldatos, C. R., Dikeos, D. G., \& Paparrigopoulos, T. J. (2003). The diagnostic validity of the Athens Insomnia Scale. Journal of psychosomatic research, 55(3), 263-267.

(33) Su, Huang. \& Chou. (2016). Effects of Destination Social Responsibility and Tourism Impacts on Residents Support for Tourism and Perceived Quality of Life. Journal of Hospitality \& Tourism Research 42(2).

(34) Timalsina, R. (2017). Factors associated with insomnia among elderly of a selected community of Lalitpur. Journal of Gerontology and Geriatric Research, 6(410), 2.

(35) Tsaras, K., Tsiantoula, M., Papathanasiou, I. V., Papagiannis, D., Chatzi, M., \& Fradelos, E. C. (2021). Predictors of Depression and Insomnia in CommunityDwelling Elderly People: A Cross-Sectional Evidence of Their Bidirectional Relationship. Cureus, 13(3).

(36) Tucker, M. A. (2020). The Value of Sleep for Optimizing Health. In Nutrition, Fitness, and Mindfulness (pp. 203215).

(37) Wang, Y. M., Chen, H. G., Song, M., Xu, S. J., Yu, L. L., Wang, L., \& Lu, L. (2016). Prevalence of insomnia and its risk factors in older individuals: a community-based study in four cities of Hebei Province, China. Sleep medicine, 19, 116-122.

(38) Weronika, W.F., Iwona, E.P. \& Bożena, M. (2016) Coexistence of Insomnia and chronic diseases in over 60 year's olds. Family Medicine \& Primary Care Review 18(3): 364-367.

(39) World health organization. (2019). Insomnia in the elderly.a review. J Clin Sleep Med. 2019; 14(6):1017-1024. 